DOI: 10.17516/1997-1397-2020-13-3-314-330

УДК 512.7

\title{
Enumeration of Bi-Commutative-AG-groupoids
}

\author{
Muhammad Rashad* \\ Imtiaz Ahmad ${ }^{\dagger}$ \\ University of Malakand \\ Chakdara Dir(L), Pakistan \\ Muhammad Shah \\ Government Collage Peshawar \\ Peshawar, Pakistan
}

A. Borumand Saeid ${ }^{\S}$

Shahid Bahonar University of Kerman Kerman, Iran

Received 29.10.2019, received in revised form 04.02.2020, accepted 09.03.2020

\begin{abstract}
In this paper, we introduce (left, right) bi-commutative AG-groupoids and provide a simple method to test whether an arbitrary AG-groupoid is bi-commutative AG-groupoid or not. We also explore some of the general properties of these AG-groupoids. Further we introduce and study some properties of ideals in these AG-groupoids and decompose left commutative AG-groupoids by defining some congruences on these AG-groupoids.
\end{abstract}

Keywords: AG-groupoid, Bi-Commutative-AG-groupoids, (left, right) commutative AG-groupoids.

Citation: M.Rashad, I.Ahmad, M.Shah, A.Borumand Saeid, Enumeration of Bi-Commutative-AGgroupoids, J. Sib. Fed. Univ. Math. Phys., 2020, 13(3), 314-330.

DOI: $10.17516 / 1997-1397-2020-13-3-314-330$.

\section{Introduction and preliminaries}

An AG-groupoid $S$ is in general a non-associative groupoid that satisfies the left invertive law,

$$
(a b) c=(c b) a \quad \forall a, b, c \in S .
$$

It is called medial if satisfies the medial property, $(a b)(c d)=(a c)(b d) \forall a, b, c, d \in S$. It is easy to prove that every AG-groupoid is medial [1]. An AG-grouoid is called an AG-monoid if it contains the left identity element. Every AG-monoid is paramedial [2], i.e., it satisfies the identity, $(a b)(c d)=(d b)(c a)$. Recently many new classes of AG-groupoids have been introduced by various researchers [3-9]. These new classes are studied in a variety of papers like for instance [10-15]. AG-groupoid is a vast field of algebra that can have almost all concepts of other algebraic structures with different characteristics and properties. A rapid research in this area can be seen on various aspects in a couple of years. AG-groupoids have a range of applications in flocks theory [1], geometry [16], topology [17], matrices [18] and in finite mathematics [19]. The structure of AG-groupoid has been strengthen by AG-rings [20,21]. Recently many varieties of ideals, $\Gamma$-ideals, bi-ideals prime ideals, semiprime ideals and quasiprime ideals have also been

\footnotetext{
*rashad@uom.edu.pk

†iahmaad@hotmail.com

¥shahmaths_problem@hotmail.com

§arsham@uk.ac.ir

(C) Siberian Federal University. All rights reserved
} 
defined and investigated by various researchers [22-25]. Fuzzification of AG-groupoids [26, 27] and other relevant concepts have also made the field interesting and valuable. All this have attracted a considerable researchers to investigate and enhance the area.

A groupoid $G$ is called left (resp. right) commutative groupoid if $G$ satisfies the identity $(a b) c=(b a) c$ (resp. $a(b c)=a(c b)) \quad \forall a, b, c \in G$ [28]. In this article, we extend the concept of these groupoids to introduce new classes of AG-groupoids as left commutative AG-groupoid or shortly an LC-AG-groupoid, a right commutative AG-groupoid or an RC-AG-groupoid, and a Bi-commutative AG-groupoid or BC-AG-groupoid. In Section 2, we properly define these notions and list some non-associative examples of these AG-groupoids to show their existence. In Subsection 2.1 and 2.2 we provide a method to verify an arbitrary AG-groupoid for LC-AGgroupoid and RC-AG-groupoid. We use the GAP software [29] and the relevant data of [29] to enumerate these new classes of AG-groupoids up to order 6 . We discuss the enumeration of these AG-groupoids in Section 3. In Section 4, we define and characterize ideals of these AG-groupoids, while in Section 5, we investigate some basic properties of these AG-groupoids and establish their relations with some of the already known AG-groupoids. We list these known subclasses of AG-groupoids with their defining identities in Tab. 1, that arise in various papers like, $[16,18,19]$ and are used in the rest of this article.

Table 1. AG-groupoid with their defining identities

\begin{tabular}{|c|c|}
\hline AG-groupoid & satisfying identity \\
\hline Left nuclear square AG-groupoid & $a^{2} \cdot b c=a^{2} b \cdot c$ \\
\hline Middle nuclear square AG-groupoid & $a b^{2} \cdot c=a \cdot b^{2} c$ \\
\hline Right nuclear square AG-groupoid & $a b \cdot c^{2}=a \cdot b c^{2}$ \\
\hline $\mathrm{T}^{1}$-AG-groupoid & $a b=c d \Rightarrow b a=d c$ \\
\hline Medial AG-groupoid & $a b \cdot c d=a c \cdot b d$ \\
\hline Paramedial AG-groupoid & $a b \cdot c d=d b \cdot c a$ \\
\hline Flexible -AG-groupoid & $a b \cdot a=a \cdot b a$ \\
\hline AG-3-band & $a \cdot a a=a a \cdot a=a$ \\
\hline Left alternative AG-groupoid & $a a \cdot b=a \cdot a b$ \\
\hline Self-dual AG-groupoid & $a \cdot b c=c \cdot b a$ \\
\hline $\mathrm{AG}^{*}$-groupoid & $a b \cdot c=b \cdot a c$ \\
\hline $\mathrm{AG}^{* *}$-groupoid & $a \cdot b c=b \cdot a c$ \\
\hline
\end{tabular}

\section{Bi-commutative-AG-groupoids and Bi-commutative AG-test}

We extend the concept of bi-commutativity of groupoid [28] to AG-groupoid and introduce the following subclasses of AG-groupoids.

Definition 1. An AG-groupoid $S$ is called

1. - a left commutative AG-groupoid (LC-AG-groupoid) if $\forall a, b, c \in S$,

$$
(a b) c=(b a) c
$$

2. - a right commutative AG-groupoid (RC-AG-groupoid) if $\forall a, b, c \in S$,

$$
a(b c)=a(c b)
$$


3. - a bi-commutative AG-groupoid (BC-AG-groupoid) if it is both LC-AG-groupoid and an $R C$-AG-groupoid.

Example 1. Let $S=\{1,2,3,4\}$. Then one can easily verify that:

(i) $(S, \cdot)$ in table (i) is an LC-AG-groupoid of order 4 and satisfies Equation 2.1,

(ii) $(S, *)$ in table $($ ii $)$ is an $R C$-AG-groupoid of order 4 that satisfies Equation 2.2 and

(iii) $(S, \circ)$ in table (iii) is BC-AG-groupoid of order 4 and satisfies both the properties of (2.1) and (2.2).

\begin{tabular}{c|cccc}
$\cdot$ & 1 & 2 & 3 & 4 \\
\hline 1 & 1 & 1 & 3 & 3 \\
2 & 1 & 1 & 4 & 3 \\
3 & 3 & 3 & 1 & 1 \\
4 & 3 & 3 & 1 & 1
\end{tabular}

\begin{tabular}{c|cccc}
$*$ & 1 & 2 & 3 & 4 \\
\hline 1 & 1 & 1 & 1 & 1 \\
2 & 1 & 1 & 1 & 3 \\
3 & 1 & 1 & 1 & 1 \\
4 & 2 & 2 & 2 & 1
\end{tabular}

\begin{tabular}{l|llll}
$\circ$ & 1 & 2 & 3 & 4 \\
\hline 1 & 1 & 2 & 2 & 2 \\
2 & 2 & 1 & 1 & 1 \\
3 & 2 & 1 & 1 & 1 \\
4 & 3 & 1 & 1 & 1
\end{tabular}

The procedure of testing a groupoid for an AG-groupoid has been explained by P. V. Protic and N. Stevanovic [24]. Here we also present a similar method to verify an arbitrary AG-groupoid for LC and RC-AG-groupoids.

\section{Left Commutative AG-groupoid Test}

We describe a procedure to test whether an arbitrary AG-groupoid $(G, \cdot)$ is an LC-AGgroupoid or not. For this we define the following binary operations:

$$
\begin{aligned}
& a \circ b=(a b) x \\
& a \star b=(b a) x
\end{aligned}
$$

Now (2.1) holds if

$$
a \circ b=a \star b
$$

or

$$
a \circ b=b \circ a
$$

To test whether an arbitrary AG-groupoid is an LC-AG-groupoid, it is necessary and sufficient to check if the operation " $\circ$ " and " $\star$ " coincide $\forall x \in G$. To this end we check the validity of Identity (2.1) or $a \circ b=a \star b$. In other words it is enough to check whether the operation $\circ$ is commutative i.e. $a \circ b=b \circ a$. The tables of the operation " $\circ$ " for any fixed $x \in G$ is obtained by multiplying a fixed element $x \in G$ by the elements of the "." table row-wise. It further gives the tables of the operation " $\star$ " if these are symmetric along the main diagonal. Hence it could easily be checked whether an arbitrary AG-groupoid is left commutative AG-groupoid or not. We illustrate this procedure with the following example.

Example 2. Check the following AG-groupoids $\left(G_{1}, \cdot\right)$ and $\left(G_{2}, \cdot\right)$ for an LC-AG-groupoid.

Table 2

\begin{tabular}{c|ccc}
$\cdot$ & 1 & 2 & 3 \\
\hline 1 & 1 & 1 & 1 \\
2 & 1 & 1 & 1 \\
3 & 2 & 2 & 2
\end{tabular}

Table 3

\begin{tabular}{l|lll}
$\cdot$ & 1 & 2 & 3 \\
\hline 1 & 1 & 2 & 3 \\
2 & 3 & 1 & 2 \\
3 & 2 & 3 & 1
\end{tabular}


We extend Tab. 2 in the way as described above. It is obvious that the tables constructed for the operation " $\circ$ " on the right of the original table are symmetric about the main diagonal and thus coincide with the " $\star$ " tables as required. Hence $\left(G_{1}, \cdot\right)$ is an LC-AG-groupoid. While in extended table for Tab. 3 is not symmetric about the main diagonal and thus $\left(G_{2}, \cdot\right)$ is not an LC-AG-groupoid.

\begin{tabular}{|c|ccc||ccc|ccc|ccc|}
\hline$\cdot$ & 1 & 2 & 3 & & 1 & & & 2 & & & 3 & \\
\hline 1 & 1 & 1 & 1 & 1 & 1 & 1 & 1 & 1 & 1 & 1 & 1 & 1 \\
2 & 1 & 1 & 1 & 1 & 1 & 1 & 1 & 1 & 1 & 1 & 1 & 1 \\
3 & 2 & 2 & 2 & 1 & 1 & 1 & 1 & 1 & 1 & 1 & 1 & 1 \\
\hline
\end{tabular}

Extended table for $\left(G_{1}, \cdot\right)$

\begin{tabular}{|l|lll||lll|lll|lll|}
\hline$\cdot$ & 1 & 2 & 3 & & 1 & & & 2 & & & 3 & \\
\hline 1 & 1 & 2 & 3 & 1 & 3 & 2 & 2 & 1 & 3 & 3 & 2 & 1 \\
2 & 3 & 1 & 2 & 2 & 1 & 3 & 3 & 2 & 1 & 1 & 3 & 2 \\
3 & 2 & 3 & 1 & 3 & 2 & 1 & 1 & 3 & 2 & 2 & 1 & 3 \\
\hline
\end{tabular}

Extended table for $\left(G_{2}, \cdot\right)$

\section{Right Commutative AG-groupoid Test}

Now, we discuss a procedure to check an AG-groupoid $(G, \cdot)$ for RC-AG-groupoid, for this we define the following two binary operations:

$$
\begin{aligned}
& a \triangleright b=a(b x) \\
& a \diamond b=a(x b)
\end{aligned}
$$

Equation (2.2) holds if,

$$
a \gg b=a \diamond b
$$

For any fixed $x \in G$, re-writing $x$-row of the ". " table as an index row of the new table and multiplying it by the elements of the index column to construct table of operation " $\diamond$ ". These extended tables are given to the right of the original table in the following example. Similarly the table for the operation " $\bigcirc$ " for any fixed $x \in G$ is obtained by taking the elements of $x$ column of the "." table as an index row of the new table and multiplying it by the elements of the index column of the original table to construct tables for the operation "ऽ", which are given downward in the extended table of the following example. If the tables for the operation " $\bigcirc$ " and " $\vee$ " coincides for all $x \in G$, then Equation (2.9) holds and the AG-groupoid is right commutative-AG-groupoid in this case.

Example 3. Check the following AG-groupoid for $R C$-AG-groupoid.

\begin{tabular}{|l|lll|}
\hline$\cdot$ & 1 & 2 & 3 \\
\hline 1 & 1 & 1 & 1 \\
2 & 1 & 1 & 1 \\
3 & 2 & 2 & 2 \\
\hline
\end{tabular}

Extend the above table in the way as described we get the extended form as follows: 


\begin{tabular}{|l|lll||lll|lll|lll|}
\hline$\cdot$ & 1 & 2 & 3 & 1 & 1 & 1 & 1 & 1 & 1 & 2 & 2 & 2 \\
\hline 1 & 1 & 1 & 1 & 1 & 1 & 1 & 1 & 1 & 1 & 1 & 1 & 1 \\
2 & 1 & 1 & 1 & 1 & 1 & 1 & 1 & 1 & 1 & 1 & 1 & 1 \\
3 & 2 & 2 & 1 & 2 & 2 & 2 & 2 & 2 & 2 & 2 & 2 & 2 \\
\hline \multicolumn{1}{r||}{} & & & 1 & 1 & 1 & 1 & 1 & 1 & 1 & 1 & 1 \\
& & & & 1 & 1 & 1 & 1 & 1 & 1 & 1 & 1 & 1 \\
& & & & 2 & 2 & 2 & 2 & 2 & 2 & 2 & 2 \\
\hline
\end{tabular}

It is clear from the extended table that the tables for the operations " $\odot$ " and " $\diamond "$ coincide for every $x \in G$, so $(G, \cdot)$ is an RC-AG-groupoid.

\section{Enumeration of BC-AG-groupoids}

Enumeration and classification of various mathematical entries is a well worked area of research in finite and pure mathematics. In abstract algebra the classification of algebraic structure is an important pre-requisite for their construction. The classification of finite simple groups is considered as one of the major intellectual achievement of twentieth century. Enumeration results can be obtained by a variety of means like; combinatorial or algebraic consideration. Non-associative structures, quasigroup and loops have been enumerated up to size 11 using combinatorial consideration and bespoke exhaustive generation software [30]. FINDER (Finite domain enumeration) [31] has been used for enumeration of IP loops up to size 13 [32]. Associative structures, semigroups and monoids have been enumerated up to size 9 and 10 respectively by constraint satisfaction techniques implemented in the Minion constraint solver with bespoke symmetry breaking provided by the computer algebra system GAP [29]. The third author of this article has implemented the same techniques under the guidance of A. Distler (the author of [33-35]) to deal the enumeration of AG-groupoids using the constraint solving techniques developed for semigroups and monoids.

Further, they provided a simple enumeration of the structures by the constraint solver and obtained a further division of the domain into a subclass of AG-groupoids using the computer algebra system GAP and were able to enumerate all AG-groupoids up to isomorphism up to size 6. They also presented enumeration for various other subclasses of AG-groupoids.

It is worth mentioning that most of the data presented in [36] has been verified by one of the reviewers of the said article with the help of Mace4 and Isofilter as has been mentioned in the acknowledgement of the said article. All this validate the enumeration and classification results for our bi-commutative AG-groupoids, as the same technique and relevant data of [36] has been used for the purpose. Further, all the tables of size up to 3 have been verified manually for our BC-AG-groupoids. In the following we describe the used algorithms with GAP commands for enumeration of our subclasses of AG-groupoid.

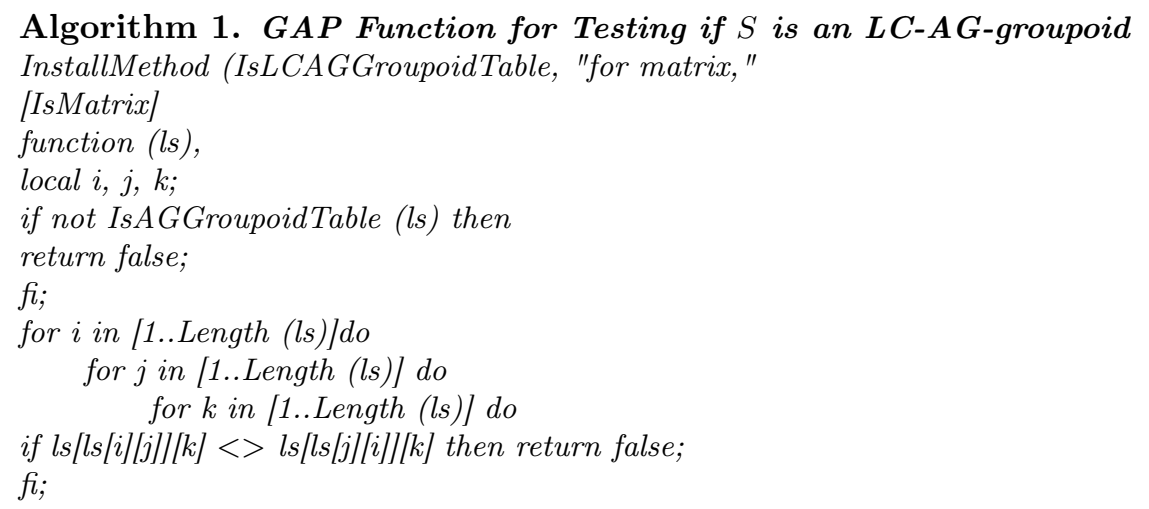


od;
$\quad$ od;
od;
return true;
end );

Algorithm 2. GAP Function for Testing if $S$ is an RC-AG-groupoid InstallMethod (IsRCAGGroupoidTable, "for matrix,"

[IsMatrix]

function (ls),

local $i, j, k$;

if not IsAGGroupoidTable (ls) then

return false;

fi;

for $i$ in $[1$...Length $(l s)]$ do

for $j$ in $[1$..Length $(l s)]$ do

for $k$ in $[1 .$. Length $(l s)]$ do

if $l s[i][l s[j][k]]<>l s[i][l s[k][j]]$ then return false;

$f i$;

od;

od;

od;

return true;

end );

Tab. 4 presents the enumeration of BC-AG-groupoids of order 3 to 6 .

Table 4. Enumeration of BC-AG-groupoids up to order 6

\begin{tabular}{|l|c|c|c|c|}
\hline Order & 3 & 4 & 5 & 6 \\
\hline \hline Total AG-groupoids & 8 & 269 & 31467 & 40097003 \\
\hline LC-AG-groupoids & 6 & 194 & 22276 & 34845724 \\
\hline RC-AG-groupoids & 2 & 52 & 1800 & 170977 \\
\hline BC-AG-groupoids & 2 & 47 & 1558 & 150977 \\
\hline
\end{tabular}

\section{Ideals in LC-AG-groupoids and RC-AG-groupoids}

In this section, we investigate ideals for LC and RC-AG-groupoids. We also characterize LC and RC-AG-groupoids by the properties of their minimal ideals. We start with the following definition and list some observations regarding ideals for LC and RC-AG-groupoids.

A subset $A$ of the AG-groupoid $S$ is a left (right) ideal of $S$ if,

$$
S A \subseteq A(A S \subseteq A)
$$

$A$ is a two sided ideal or simply an ideal of $S$ if it is both left and right ideal of $S$.

Remark 1 ([24]). If $S$ is an AG-groupoid and $a \in S$, then by the Identity (1.1), it follows that:

$$
(a S) S=\cup_{x, y \in S}(a x) y=\cup_{x, y \in S}(y x) a \subseteq S a .
$$

From this we conclude that $(A S) S \subseteq S A$.

Further we have the following remarks. 
Remark 2. If $S$ is an AG-groupoid with left identity e and $a \in S$, then by the medial property and Identity (2.2), it follows that:

$$
S(a S)=\cup_{x, y \in S} x(a y)=\underset{x, y \in S}{\cup}(e x)(a y)=\cup_{x, y \in S}(e a)(x y) \subseteq a S .
$$

In general for any $A \subseteq S$ we conclude that $S(A S) \subseteq A S$.

Remark 3. If $S$ is an $L C$-AG-groupoid and $a \in S$, then by left invertive law and (2.1), it follows that:

$$
(S a) S=\underset{x, y \in S}{\cup}(x a) y=\underset{x, y \in S}{\cup}(a x) y=\underset{x, y \in S}{\cup}(y x) a \subseteq S a .
$$

Thus for any $A \subseteq S$ we conclude that $(S A) S \subseteq S A$.

Remark 4. If $S$ is an RC-AG-groupoid with left identity e and $a \in S$, then by left invertive law and (2.2), it follows that:

$$
\begin{aligned}
S(S a) & =\underset{x, y \in S}{\cup} x(y a)=\underset{x, y \in S}{\cup}(e x)(y a)= \\
& =\underset{x, y \in S}{\cup}(e x)(a y)=\underset{x, y \in S}{\cup}(e a)(x y) \subseteq a S .
\end{aligned}
$$

Hence in general $S(S A) \subseteq A S$ for $A \subseteq S$.

Remark 5. If $S$ is an RC-AG-groupoid with left identity e and $a \in S$, then by medial law and by Identity (2.1), it follows that:

$$
\begin{aligned}
(S a) S & =\cup_{x, y \in S}(x a) y=\underset{x, y \in S}{\cup}(x a)(e y)=\underset{x, y \in S}{\cup}(x a)(y e)= \\
& =\cup_{x, y \in S}(x y)(a e)=\cup_{x, y \in S}(x y)(e a) \subseteq S a .
\end{aligned}
$$

Thus $(S A) S \subseteq S A$ for $A \subseteq S$.

Definition 2 ([24]). Let $S$ be an $A G$-groupoid and $A, B \subseteq S$, than $A$ and $B$ are right (left) connected sets if $A S \subseteq B$ and $B S \subseteq A(S A \subseteq B \quad \mathcal{E} S B \subseteq A)$.

Example 4. Let $S=\{1,2,3,4\}$ be an AG-groupoid given by the following table

\begin{tabular}{|c|cccc|}
\hline$\cdot$ & 1 & 2 & 3 & 4 \\
\hline 1 & 1 & 1 & 1 & 1 \\
2 & 1 & 1 & 1 & 1 \\
3 & 2 & 1 & 1 & 1 \\
4 & 2 & 1 & 2 & 1 \\
\hline
\end{tabular}

Now and $A=\{1,2,3\}$ and $B=\{1,2,4\}$ be two subsets of $S$. Then clearly $A S \subseteq B$ and $B S \subseteq A$ also $S A \subseteq B$ and $S B \subseteq A$. Thus $A$ and $B$ are left and right connected and hence are connected.

Remark 6. If $L$ is a left and $R$ is a right ideal of an $L C$-AG-groupoid $S$, then by left invertive law and Identity (4.1), we have

$$
(L R) S=(S R) L=(R S) L \subseteq R L \text { and }(R L) S=(S L) R \subseteq L R .
$$

It follows that $L R$ and $R L$ are right connected sets.

Proposition 1. Let $S$ be an $L C$-AG-groupoid. Then for each $a \in S$ the set $a \cup a S$ and $a S \cup S a$ are right connected sets. 
Proof. If $a \in S$, then by Remarks (1) and (3), we have

$$
(a \cup S a) S=a S \cup(S a) S \subseteq a S \cup S a,
$$

also,

$$
(a S \cup S a) S=(a S) S \cup(S a) S \subseteq S a \cup S a \subseteq a \cup S a .
$$

Hence the result follows.

Theorem 1. Let $S$ be an LC-AG-groupoid. Then for each $a \in S$ the set $a \cup a S \cup S a$ is right ideal of $S$.

Proof. Let $a \in S$, then by Remarks $1-3$, we have

$$
\begin{aligned}
(a \cup a S \cup S a) S & =a S \cup(a S) S \cup(S a) S \subseteq a S \cup S a \cup S a \\
& \subseteq a S \cup S a \subseteq a \cup a S \cup S a .
\end{aligned}
$$

Hence $(a \cup a S \cup S a) S$ is right ideal of $S$.

Theorem 2. Let $S$ be an $R C$-AG-groupoid with left identity $e$. Then for each a $\in S$ the set $J(a)=a \cup a S \cup S a$ is the minimal (two sided) ideal of $S$ containing a.

Proof. By Remarks (2) and (4), we have

$$
S(a \cup a S \cup S a)=S a \cup S(a S) \cup S(S a) \subseteq S a \cup a S \cup a S \subseteq S a \cup a S \subseteq a \cup a S \cup S a .
$$

Thus $J(a)$ is a left ideal. Now again by Remarks (1) and (3), we have

$$
(a \cup a S \cup S a) S=a S \cup(a S) S \cup(S a) S \subseteq a S \cup S a \cup S a \subseteq a S \cup S a \subseteq a \cup a S \cup S a .
$$

Thus $J(a)$ is a right ideal, and hence it is a two sided ideal or simply an ideal of $S$. If $J$ is an ideal of $S$ and $a \in J$, then

$$
J(a)=a \cup(a S \cup S a) \subseteq J \cup(J S \cup S J) \subseteq J \cup(J \cup J) \subseteq J \Rightarrow J(a) \subseteq J .
$$

Hence the result follows.

Theorem 3. If $S$ is an $R C$-AG-groupoid with left identity $e$, then for $a \in S$ the sets a $(S a)$ and $(a S) a$ are ideals of $S$. If $a \in a(S a)$ (resp. $a \in(a S) a)$, then $a(S a)($ resp. $(a S) a)$ is a minimal ideal generated by $a$. Further if $a \in(a(S a) \cap(a S) a)$, then $(a S) a=a(S a)$ and it is minimal ideal generated by $a$.

Proof. If $a \in S$, then by the medial law, left invertive law and (2.2), we have

$$
\begin{aligned}
S(a(S a)) & =\cup_{x, y \in S} x(a(y a))=\cup_{x, y \in S}(e x)(a(y a))=\cup_{x, y \in S}(e a)(x(y a))= \\
& =\bigcup_{x, y \in S} a(x(a y))=\bigcup_{x, y \in S} a((a y) x)=\cup_{x, y \in S} a((x y) a) \subseteq a(S a) .
\end{aligned}
$$

Similarly, by paramedial, medial, left invertive laws and (2.2), we have

$$
\begin{aligned}
(a(S a)) S= & \cup_{x, y \in S}(a(x a)) y=\cup_{x, y \in S}(y(x a)) a=\cup_{x, y \in S}(y(a x)) a= \\
= & \bigcup_{x, y \in S}(y(a x))(e a)=\cup_{x, y \in S}(y(a x))(a e)=\underset{x, y \in S}{\cup}(e(a) x)(a y)= \\
= & \bigcup_{x, y \in S}(e a)((a x) y)=\bigcup_{x, y \in S} a((y x) a) \subseteq a(S a) . \\
& -321-
\end{aligned}
$$


Hence, $a(S a)$ is an ideal of $S$. Now, again using the paramedial and left invertive laws and the Identity (2.2), we have

$$
\begin{aligned}
S(((a S) a)) & =\cup_{x, y \in S} x((a y) a)=\underset{x, y \in S}{\cup} x(a(a y))=\underset{x, y \in S}{\cup}(e x)(a(a y))= \\
& =\bigcup_{x, y \in S}(e a)(x(a y))=\cup_{x, y \in S}(e a)((a y) x)=\cup_{x, y \in S}(e a)((x y) a)= \\
& =\bigcup_{x, y \in S}(a a)((x y) e)=\cup_{x, y \in S}(a(x y))(a e)=\cup_{x, y \in S}(a(x y))(e a)= \\
& =\bigcup_{x, y \in S}(a(x y)) a \subseteq(a S) a \Rightarrow S(((a S) a)) \subseteq(a S) a .
\end{aligned}
$$

Similarly,

$$
\begin{aligned}
((a S) a) S & =\cup_{x, y \in S}((a x) a) y=\cup_{x, y \in S}(y a)(a x)=\cup_{x, y \in S}(y a)(x a)=\underset{x, y \in S}{\cup}(y x)(a a)= \\
& =\cup_{x, y \in S}(e(y x))(a a)=\cup_{x, y \in S}(a(y x))(a e)=\cup_{x, y \in S}(a(y x))(e a) \\
& \subseteq(a S) a \Rightarrow((a S) a) S \subseteq(a S) a .
\end{aligned}
$$

Hence $(a S) a$ and $a(S a)$ are ideals of $S$. If $A$ is an ideal on $S$, then for every $a \in A$ we have $(a S) a \subseteq$ $A$ and $a(S a) \subseteq A$, clearly. If $a \in A \cap(a S) a($ resp. $a \in A \cap a(S a))$, then $(a S) a$ (resp. $a(S a))$ is a minimal ideal generated by $a$. If $a \in A \cap(a S) a \cap a(S a)$, then by minimality, it follows that $(a S) a=a(S a)$. Clearly, for each $a \in S$ it holds that $(a S) a \subseteq S a$ and $a(S a) \subseteq S a$.

\section{Characterization of BC-AG-groupoids}

In this section, we discuss the relations of BC-AG-groupoid with some already known subclasses of AG-groupoids. We start with the following results which proves that every $\mathrm{AG}^{*}$ groupoid is RC-AG-groupoid, but the converse is not always true as illustrated in Example 5. The Example 6 also shows that every LC-AG-groupoid may not be an AG*-groupoid.

Theorem 4. Every $A G^{*}$-groupoid is $R C$-AG-groupoid.

Proof. Let $S$ be an $\mathrm{AG}^{*}$-groupoid, and $a, b, c \in S$. Then

$$
a(b c)=(b a) c=(c a) b=a(c b) \Rightarrow a(b c)=a(c b) .
$$

Hence $S$ is RC-AG-groupoid.

Example 5. Let $S=\{1,2,3\}$ be an $R C$-AG-groupoid with the following table. Then $(S, *)$ is not an $A G^{*}$-groupoid since $(1 * 1) * 1 \neq 1 *(1 * 1)$.

\begin{tabular}{l|lll}
$*$ & 1 & 2 & 3 \\
\hline 1 & 2 & 2 & 2 \\
2 & 3 & 3 & 3 \\
3 & 3 & 3 & 3
\end{tabular}

Example 6. Let $S=\{1,2,3,4,5,6\}$. Then it is easy to verify that $S$ is an $A G^{*}$-groupoid, but not an LC-AG-groupoid as clearly, $(1 * 2) * 1 \neq(2 * 1) * 1$.

\begin{tabular}{c|cccccc}
$*$ & 1 & 2 & 3 & 4 & 5 & 6 \\
\hline 1 & 3 & 4 & 5 & 5 & 5 & 5 \\
2 & 3 & 4 & 6 & 6 & 5 & 5 \\
3 & 5 & 5 & 5 & 5 & 5 & 5 \\
4 & 6 & 6 & 5 & 5 & 5 & 5 \\
5 & 5 & 5 & 5 & 5 & 5 & 5 \\
6 & 5 & 5 & 5 & 5 & 5 & 5
\end{tabular}


Now, we prove the following:

Theorem 5. Every $L C$-A $G^{*}$-groupoid is a semigroup.

Proof. Let $S$ be an LC-AG*-groupoid, then for every $a, b, c \in S$

$$
a b \cdot c=b a \cdot c=a \cdot b c \Rightarrow a b \cdot c=a \cdot b c .
$$

Thus $S$ is semigroup.

The converse of the above theorem is not true as shown in the following example.

Example 7. Let $S=\{1,2,3,4,5,6\}$. Then $(S, *)$ with the given table is a semigroup. Clearly $S$ is neither $L C$-AG-groupoid nor $A G^{*}$-groupoid as, $(0 * 1) * 0 \neq 1 *(0 * 0)$ and $(0 * 1) * 0 \neq(1 * 0) * 0$.

\begin{tabular}{c|cccccc}
$*$ & 0 & 1 & 2 & 3 & 4 & 5 \\
\hline 0 & 2 & 3 & 2 & 2 & 5 & 2 \\
1 & 4 & 2 & 2 & 2 & 2 & 2 \\
2 & 2 & 2 & 2 & 2 & 2 & 2 \\
3 & 5 & 2 & 2 & 2 & 2 & 2 \\
4 & 2 & 2 & 2 & 2 & 2 & 2 \\
5 & 2 & 2 & 2 & 2 & 2 & 2
\end{tabular}

An element $a$ of an AG-groupoid $S$ is called left cancellative if $a b=a c \Rightarrow b=c$, right cancellative and cancellative elements are defined analogously.

Theorem 6. Let $S$ be an LC-AG-groupoid. Then $S$ is a commutative semigroup if it has a right cancellative element.

Proof. Let $x$ be a a right cancellative element of an LC-AG-groupoid $S$, and $a, b \in S$. Then

$$
(a b) x=(b a) x \Rightarrow a b=b a .
$$

Thus $S$ is commutative, but commutativity implies associativity in AG-groupoids. Hence $S$ is a commutative semigroup.

Theorem 7. Every LC-AG-groupoid is paramedial AG-groupoid.

Proof. Let $S$ be a LC-AG-groupoid, and $a, b, c, d \in S$. Then

$$
\begin{aligned}
(a b)(c d) & =(b a)(c d)=((c d) a) b=((d c) a) b=((a c) d) b= \\
& =((c a) d) b=(b d)(c a)=(d b)(c a) \\
\Rightarrow(a b)(c d) & =(d b)(c a) .
\end{aligned}
$$

Hence $S$ is a paramedial AG-groupoid.

Example 8. The following is an example of $R C$-AG-groupoid of order 4 that is not paramedial AG-groupoid.

\begin{tabular}{l|llll}
$*$ & 1 & 2 & 3 & 4 \\
\hline 1 & 1 & 3 & 1 & 1 \\
2 & 4 & 4 & 4 & 4 \\
3 & 1 & 3 & 1 & 1 \\
4 & 3 & 1 & 3 & 3
\end{tabular}

Theorem 8. Every LC-AG-groupoid is left nuclear square AG-groupoid. 
Proof. Let $S$ be a LC-AG-groupoid, and $a, b, c \in S$. Then

$$
\begin{aligned}
a^{2}(b c)= & (a a)(b c)=((b c) a) a=((c b) a) a=((a b) c) a= \\
& =(c(a b)) a=(a(a b)) c=(a b) a) c=((b a) a) c= \\
= & ((a a) b) c=\left(a^{2} b\right) c \Rightarrow a^{2}(b c)=\left(a^{2} b\right) c .
\end{aligned}
$$

Hence $S$ is left nuclear square AG-groupoid.

The following counterexample shows that neither $\mathrm{AG}^{* *}$-groupoid nor BC-AG-groupoid is nuclear square AG-groupoid. However, both these properties jointly gives the desired relation as given in the following theorem.

\section{Example 9.}

(i) $A G^{* *}$-groupoid that is not a nuclear square $A G$-groupoid as $(3 * 3) * 3^{2} \neq 3 *\left(3 * 3^{2}\right)$.

(ii) BC-AG-groupoid that is not nuclear square AG-groupoid as $(3 * 3) * 3^{2} \neq 3 *\left(3 * 3^{2}\right)$.

\begin{tabular}{l|lll}
$*$ & 1 & 2 & 3 \\
\hline 1 & 1 & 1 & 1 \\
2 & 1 & 1 & 1 \\
3 & 1 & 2 & 2
\end{tabular}

(i)

\begin{tabular}{l|lll}
$*$ & 1 & 2 & 3 \\
\hline 1 & 1 & 1 & 1 \\
2 & 1 & 1 & 1 \\
3 & 2 & 2 & 1
\end{tabular}

(ii)

Theorem 9. Let $S$ be a $B C$ - $A G^{* *}$-groupoid. Then the following assertions are equivalent.

(i) $S$ is left nuclear square AG-groupoid;

(ii) $S$ is middle nuclear square AG-groupoid;

(iii) $S$ is right nuclear square AG-groupoid.

Proof. Let $S$ be a BC-AG**-groupoid. Then

$(i) \Rightarrow(i i)$. Assume $(i)$ holds, let $a, b, c \in S$. Then

$$
a\left(b^{2} c\right)=b^{2}(a c)=b^{2}(c a)=\left(b^{2} c\right) a=\left(c b^{2}\right) a=\left(a b^{2}\right) c \Rightarrow a\left(b^{2} c\right)=\left(a b^{2}\right) c .
$$

Thus $S$ is middle nuclear square AG-groupoid.

$($ ii $) \Rightarrow($ iii $)$. Assume $(i i)$ holds, let $a, b, c \in S$. Then

$$
a\left(b c^{2}\right)=b\left(a c^{2}\right)=b\left(c^{2} a\right)=\left(b c^{2}\right) a=\left(c^{2} b\right) a=(a b) c^{2} \Rightarrow a\left(b c^{2}\right)=(a b) c^{2} .
$$

Finally we show,

$($ iii $) \Rightarrow(i)$. Assume (iii) holds, and $a, b, c \in S$. Then

$$
a^{2}(b c)=b\left(a^{2} c\right)=b\left(c a^{2}\right)=(b c) a^{2}=(c b) a^{2}=\left(a^{2} b\right) c \Rightarrow a^{2}(b c)=\left(a^{2} b\right) c .
$$

which proves $(i)$. Hence the theorem is proved.

Now, we give an example of left alternative AG-groupoid and BC-AG-groupoid that are not flexible AG-groupoid.

Example 10. (i) The AG-groupoid in Table (i) below, is left alternative but not flexible AGgroupoid.

(ii) The AG-groupoid in Table (ii) is BC-AG-groupoid, but not flexible AG-groupoid. 


\begin{tabular}{l|llll}
$*$ & 1 & 2 & 3 & 4 \\
\hline 1 & 1 & 1 & 1 & 1 \\
2 & 1 & 1 & 1 & 3 \\
3 & 1 & 4 & 1 & 1 \\
4 & 1 & 1 & 2 & 1
\end{tabular}

(i)

\begin{tabular}{l|llll}
$*$ & 1 & 2 & 3 & 4 \\
\hline 1 & 1 & 1 & 1 & 1 \\
2 & 1 & 1 & 1 & 1 \\
3 & 1 & 1 & 2 & 1 \\
4 & 2 & 2 & 1 & 1
\end{tabular}

(ii)

However, we have the following:

Theorem 10. Every BC-AG-groupoid is left alternative AG-groupoid if and only if it is flexible AG-groupoid.

Proof. Let $S$ be a BC-AG-groupoid satisfying the left alternative AG-groupoid property, and let $a, b \in S$. Then

$$
(a b) a=(b a) a=(a a) b=a(a b)=a(b a) \Rightarrow(a b) a=a(b a) .
$$

Hence $S$ is flexible AG-groupoid.

Conversely let $S$ be a BC-AG-groupoid satisfying the flexible AG-groupoid property, then for $a, b \in S$, we have

$$
(a a) b=(b a) a=(a b) a=a(b a)=a(a b) \Rightarrow(a a) b=a(a b) .
$$

Hence $S$ is left alternative AG-groupoid.

One can easily verify in the following tables that neither $T^{1}$-AG-groupoid nor BC-AGgroupoid is self-dual AG-groupoid.

Example 11. 1. The AG-groupoid given in Table (i) is $T^{1}$-AG-groupoid but not self-dual AG-groupoid, while the AG-groupoid in Table (ii) is BC-AG-groupoid but not self-dual AG-groupoid.

\begin{tabular}{l|lll}
$*$ & 1 & 2 & 3 \\
\hline 1 & 1 & 1 & 1 \\
2 & 1 & 1 & 3 \\
3 & 1 & 2 & 1
\end{tabular}

\begin{tabular}{c|cccc}
$*$ & 1 & 2 & 3 & 4 \\
\hline 1 & 1 & 1 & 1 & 1 \\
2 & 1 & 1 & 1 & 1 \\
3 & 1 & 1 & 1 & 2 \\
4 & 1 & 1 & 1 & 4
\end{tabular}

However, we prove the following:

Theorem 11. Every $T^{1}-A G$-groupoid $S$ is self-dual AG-groupoid, if any of the following holds.

(i) $S$ is LC-AG-groupoid.

(ii) $S$ is RC-AG-groupoid.

Proof. Let $S$ be a $\mathrm{T}^{1}$-AG-groupoid, and let $a, b, c \in S$.

(i) If $S$ is LC-AG-groupoid, then

$$
(b c) a=(c b) a=(a b) c=(b a) c \Rightarrow a(b c)=c(b a) .
$$

(ii) Again, if $S$ is RC-AG-groupoid, then

$$
a(b c)=a(c b) \Rightarrow(b c) a=(c b) a=(a b) c \Rightarrow a(b c)=c(a b)=c(b a) .
$$


Hence $S$ is self- dual AG-groupoid in each case and the theorem is proved.

Theorem 12. Every BC-AG-3-band is commutative semigroup.

Proof. Let $S$ be BC-AG-3-band, and let $a, b \in S$. Then

$$
\begin{aligned}
a b & =(a(a a))(b(b b))=(a b)((a a)(b b))=(b a)((a a)(b b))= \\
& =(b a)((a b)(a b))=(b a)((b a)(a b))=(b a)((b a)(b a))= \\
& =(b a)((b b)(a a))=(b(b b))(a(a a))=b a \Rightarrow a b=b a .
\end{aligned}
$$

Thus $S$ is commutative and hence is associative. Equivalently $S$ is commutative semigroup.

\section{Congruences on LC-AG-groupoid}

Congruence on various subclasses of AG-groupoids are defined in various papers [23, 37, 38]. In this section we discuss some congruence on LC-AG-groupoids. It is observed that if $S$ is an LC-AG-groupoid and $E_{S} \neq \emptyset$, where $E_{S}$ is the collection of all idempotents of $S$ then by medial law, definition of LC-AG-groupoid and repeated use of the left invertive law, $E(S)$ is semilattice, that is for any $e, f \in E_{S} \neq \emptyset$ :

$$
\begin{aligned}
& e f=(e e)(f f)=(e f)(e f)=(f e)(e f)=((e f) e) f= \\
& =((f e) e) f=((e e) f) f=(f f)(e e)=f e \Rightarrow e f=f e .
\end{aligned}
$$

This implies that $E_{S}$ is commutative. Moreover, $\forall a, b \in S$ and $e \in E_{S}$, we have

$$
\begin{aligned}
e(a b) & =(e a)(e b)=((e b) a) e=((b e) a) e=((a e) b) e=((e a) b) e=(b(e a)) e= \\
& =(e(e a)) b=((a e) e) b=((e e) a) b=(e a) b \Rightarrow e(a b)=(e a) b .
\end{aligned}
$$

Thus $\forall a, b \in S$ and $e \in E_{S}$, as a consequences of the above we have the following.

Proposition 2. Let $S$ be an $L C$-AG-groupoid. Then $E_{S}$ is a semilattice.

Example 12. $L C$-AG-band of order 4 that is a semilattice.

\begin{tabular}{l|llll}
$*$ & 1 & 2 & 3 & 4 \\
\hline 1 & 1 & 1 & 1 & 1 \\
2 & 1 & 2 & 2 & 2 \\
3 & 1 & 2 & 3 & 3 \\
4 & 1 & 2 & 3 & 4
\end{tabular}

Furthermore, in Example 13, $E_{S}=\{1,3\} \subseteq S$ is a semilattice.

Theorem 13. Let $(S, \cdot)$ be an $L C$-AG-groupoid such that $E_{S} \neq \phi$, and $\eta$ be a relation defined on $S$ as

$$
\eta=\left\{(a, b) \in S,(x e) a=(y e) b \text { for every } e \in E_{S} \text { and } x, y \in(S, \cdot)\right\} .
$$

Then $\eta$ is a congruence on $S$.

Proof. Given that $S$ is an LC-AG-groupoid and $E_{S}$ is the set of all idempotent elements in $S$. A relation $\eta$ is defined on $S$ as,

$$
\eta=\left\{(a, b) \in S,(x e) a=(y e) b \text { for every } e \in E_{S}\right\}
$$


First, we show that $\eta$ is equivalence relation on $S$, for this we show that $\eta$ is reflexive, symmetric and transitive relation. Clearly $\eta$ is reflexive as for any $a, x \in S(x e) a=(x e) a \Rightarrow a \eta b$. Let $a \eta b$, then $(x e) a=(y e) b \Leftrightarrow(y e) b=(x e) a \Leftrightarrow b \eta a$. Hence $\eta$ is symmetric. Now, for transitivity let $a \eta b$ and $b \eta c$. Then $(x e) a=(y e) b$ and $(y e) b=(z e) c$ for some $x, y, z \in S \Leftrightarrow(x e) a=(y e) b=(z e) c \Leftrightarrow$ $(x e) a=(z e) c \Leftrightarrow a \eta c$. Hence $\eta$ is transitive. Therefore $\eta$ is an equivalence relation on $S$. Now, we show that $\eta$ is compatible. First we show that $\eta$ is right compatible, for this let $a, b, c$ and $x, y, z$ are elements of $S$, then using left invertive, medial laws, definition of LC-AG-groupoid and Theorem (7), we get

$$
\begin{array}{ccc}
a \eta b \Rightarrow(x e) a=(y e) b & \Rightarrow & ((x e) a) c=((y e) b) c \\
\Rightarrow(c a)(x e)=(c b)(y e) & \Rightarrow & (c x)(a e)=(c y)(b e) \\
\Rightarrow(e x)(a c)=(e y)(b c) & \Rightarrow & (x e)(a c)=(y e)(b c) \\
\Rightarrow a \eta b & \Rightarrow a c \eta b c .
\end{array}
$$

Therefore $\eta$ is right compatible. Similarly, it is easy to show that $\eta$ is left compatible. Hence $\eta$ is compatible and therefore $\eta$ is a congruence on $S$.

Theorem 14. Let $S$ be an $L C$-AG-groupoid and $E_{S} \neq \emptyset$. Let $\rho$ be a relation on $S$ defined as, $\rho=\left\{(a, b) \in S\right.$, ea $=e b$ for every $\left.e \in E_{S}\right\}$. Then $\rho$ is a congruence on $S$.

Proof. Let $S$ be an LC-AG-groupoid and $E_{S}$ denotes the set of all idempotent elements in $S$. Assume that $E_{S} \neq \emptyset$. A relation $\rho$ is defined on $S$ as,

$$
\rho=\left\{(a, b) \in S, e a=e b \text { for every } e \in E_{S}\right\} .
$$

Now, we show that $\rho$ is an equivalence relation on $S$. Obviously $\rho$ is reflexive, as for any $a \in S$ and $e \in E_{S}$ we have $e a=e a \Rightarrow a \rho b$. Let $a \rho b \Leftrightarrow e a=e b \Leftrightarrow e b=e a \Leftrightarrow b \rho a$. Hence $\rho$ is symmetric. Now, let $a \rho b$ and $b \rho c \Leftrightarrow e a=e b$ and $e b=e c$ for some $a, b \in S \Leftrightarrow e a=e b=e c \Leftrightarrow e a=e c \Leftrightarrow a \rho c$. Hence $\rho$ is transitive. Therefore $\rho$ is an equivalence relation on $S$. Next we show that $\rho$ is compatible.

$\rho$ is right compatible:

$$
\begin{aligned}
a \rho b \Leftrightarrow e a & =e b \Leftrightarrow(e a) c=(e b) c \\
\Leftrightarrow(c a) e & =(c b) e \Leftrightarrow(c a)(e e)=(c b)(e e) \\
\Leftrightarrow(a c)(e e) & =(b c)(e e) \Leftrightarrow(a e)(c e)=(b e)(c e) \\
\Leftrightarrow(e a)(c e) & =(e b)(c e) \Leftrightarrow((c e) a) e=((c e) b) e \\
\Leftrightarrow((e c) a) e & =((e c) b) e \Leftrightarrow((a c) e) e=((b c) e) e \\
\Leftrightarrow(e e)(a c) & =(e e)(b c) \Leftrightarrow e(a c)=e(b c) \\
a \rho b & \Leftrightarrow a c \rho b c .
\end{aligned}
$$

Hence $\rho$ is right compatible. Similarly $\rho$ is left compatible. Hence $\rho$ is compatible and therefore is a congruence on $S$.

Example 13. Let $S=\{1,2,3,4\}$. Then $(S, \cdot)$ with the following table is LC-AG-groupoid.

\begin{tabular}{l|llll}
$\cdot$ & 1 & 2 & 3 & 4 \\
\hline 1 & 1 & 1 & 1 & 1 \\
2 & 1 & 1 & 1 & 1 \\
3 & 1 & 1 & 3 & 1 \\
4 & 1 & 2 & 1 & 1
\end{tabular}

and $E_{S}=\{1,3\}$ define a relation $\rho$ as $a \rho b \Leftrightarrow e a=e b, \forall a, b \in S$ and $e \in E_{S}$, we have

$$
\rho=\{(1,1),(1,2),(1,4),(2,1),(2,2),(2,4),(3,3),(4,1),(4,2),(4,4)\} .
$$

Clearly $\rho$ is equivalence relation and also left and right compatible, hence is a congruence on $S$. 


\section{Conclusion}

In this article, we have introduced some new classes of AG-groupoids that are RC-AGgroupoid, LC-AG-groupoid and BC-AG-groupoid. We have provided various examples generated by GAP for the existence of these subclasses. Enumeration of these classes has also been done up to order 6 . We also introduced a procedure to verify an arbitrary AG-groupoid for these classes and proved some basic results for these newly introduced classes like; every $\mathrm{AG}^{*}$-groupoid is RCAGgroupoid, every LC-AG*-groupoid is semigroup and in general LC-AG-groupoid is semigroup only if, it has a right cancellative element or has a left identity element. We have investigated that BC-AG*-groupoid is nuclear square AG-groupoid and is left alternative if and only if it is flexible. We also investigated ideals in these classes. Some congruences have also been defined on these subclasses.

Acknowledgement. We are tankful to Professor Petar Markovic for improving this article. The authors are extremely grateful to the editor and the referees for their valuable comments and helpful suggestions which help to improve the presentation of this paper.

This research is financially supported by Government of Pakistan through HEC funded project NRPU-3509.

\section{References}

[1] M.Naseeruddin, Some studies on almost semigroups and flocks, PhD thesis, The Aligarh Muslim University, India, 1970.

[2] J.R.Cho, J.Jezek, T.Kepka, Paramedial Groupoids, Czechoslovak Mathematical Journal, 49(1996), no. 124, 277-290. DOI: 10.1023/A:1022448218116

[3] M.Khan, Faisal, V.Amjad, On some classes of Abel-Grassmann's groupoids, J. Adv. Res. Pure Math., 3(2011), no. 4, 109-119.

[4] M.Rashad, I.Ahmad, M.Shah, Amanullah, Stein AG-groupoids, Sindh University Research Journal (Science Series), 48(2016), no. 3, 679-684.

[5] M.Shah, I.Ahmad, A.Ali, Discovery of new classes of AG-groupoids, Research Journal of Recent Sciences, 1(2012), no. 11, 47-49.

[6] M.Shah, I.Ahmad, A.Ali, On introduction of new classes of AG-groupoids, Research Journal of Recent Sciences, 2(2013), no. 1, 67-70.

[7] M.Iqbal, I.Ahmad, Ideals in CA-AG-groupoids, Indian J. Pure Appl. Math., 49(2018), no. 2, 265-284. DOI: 10.1007/s13226-018-0268-5

[8] I.Ahmad, Iftikhar Ahmad, M. Rashad, A study of anti-commutativity in AG-groupoids, Punjab Univ. J. Math., 48(2016), no. 1, 99-109.

[9] M.Rashad, I.Ahmad, Amanullah, M. Shah, A study on Cheban Abel-Grassmann's groupoids, Punjab Univ. J. Math., 51(2019), no. 2, 79-90.

[10] I.Ahmad, M.Rashad, M.Shah, Some Properties of AG*-groupoid, Research Journal of Recent Sciences, 2(2013), no. 4, 91-93.

[11] I.Ahmad, M.Rashad, M.Shah, Some new result on $\mathrm{T}^{1}, \mathrm{~T}^{2}$ and $\mathrm{T}^{4}$-AG-groupoids, Research Journal of Recent Sciences, 2(2013), no. 3, 64-66. 
[12] M.Rashad, I.Ahmad, Amanullah, M.Shah, On relations between right alternative and nuclear square AG-groupoids, Int. Math. Forum, 8(2013), no. 5-8, 237-243.

DOI: $10.12988 /$ imf.2013.13024

[13] M.Rashad, I.Ahmad, M.Shah, Left transitive AG-groupoids, Sindh University Research Journal (Science Series), 46(2014), no. 4, 547-552.

[14] M.Iqbal, I.Ahmad, Some Congruences on CA-AG-groupoids, Punjab University. J. Math., $\mathbf{5 1}(2019)$, no. $3,71-87$.

[15] M.Rashad, I.Ahmad, A note on unar LA-semigroup, Punjab Univ. J. Math., 50(2018), no. 3, $113-121$.

[16] Muhammad Shah, A theoretical and computational investigation of AG-groups, $\mathrm{PhD}$ thesis, Quaid-i-Azam University Islamabad, 2012.

[17] N.Bobeica, Liubomir Chiriac, On toplogical AG-groupoids and paramedial quasigroups with multiple identities, ROMAI Journal, 6(2010), no. 1, 5-14.

[18] Amanullah, M.Rashad, I.Ahmad, Abel Grassmann's Groupoids of Modulo Matrices, Mehran University research Journal of Engineering and Technology, 35(2016), no. 1, 63-70.

[19] Amanullah, M.Rashad, I.Ahmad, M.Shah, On Modulo AG-groupoids, Journal of Advances in Mathematics, 8(2014), no. 3, 1606-1613.

[20] T.Shah, Inayat Ur Rahman, On LA-rings of finitely nonzero function, Int. J. Contemp. Math. Sciences, 5(2010), no. 5, 209-222.

[21] T.Shah, M.Raees, Gohar Ali, On LA-Modules, Int. J. Contemp. Math. Sciences, 6(2011), no. $21,999-1006$.

[22] Q.Mushtaq, M.Khan, Ideals in AG-band and $\mathrm{AG}^{*}$-groupoids, Quasigroups and Related system, 14(2006), 207-215.

[23] P.V.Protic, Congruences on an inverse $\mathrm{AG}^{* *}$-groupoid via the natural partial order, Quasigroups and related system, 17(2009), no. 2, 283-290.

[24] P.V.Protic, N.Stevanovic, AG-test and some general properties of AG-groupoids, PU.M.A. Pure Mathematics and Applications, 6(1995), no. 4, 371-383.

[25] T.Shah, Inayat Ur Rahman, On $\Gamma$-ideals and $\Gamma$-bi-ideals in $\Gamma$-AG-groupoids, International journal of Algebra, 4(2010), no. 6, 267-276.

[26] A.Khan, M.Shabir, Young Bae Jun, Generalized fuzzy Abel Grassmann's Groupoids, International Journal of Fuzzy Systems, 12(2010), no. 4, 340-349.

[27] Asghar Khan, Young Bae Jun, Tahir Mahmmod, Generalized fuzzy interior ideals in Abel Grassmann's Groupoids, International journal of Mathematics and Mathematical Sciences, 2010, Article ID 838392. DOI: 10.1155/2010/838392

[28] V.Celakoska-Jordanova, Biljiana Janeva, Free left commutative Groupoids, www.arXiv.org.

[29] GAP: Groups Algorithm and Programming, Version 4.4.12, 2008.

[30] B.D.McKay, I.M.Wanless, On the number of Latin squares, Ann. Combin., 9(2005), 335-344.

[31] J.Slaney, FINDER, Notes and Guide, Center of Information Science and Research, Australian National University, 1995. 
[32] A.Ali, J.Slaney, Counting loops with the inverse property image, Quasigroups and Related System, 16(2008), no. 1, 13-16.

[33] A.Distler, T.Kelsey, The monoids of order eight and nine, In Proc. of AISC 2008, vol. 5144 of LNCS, Springer, 2008, 61-76.

[34] A.Distler, T.Kelsey, The monoids of order eight and nine and ten, Ann. Math. Artif. Intell., 56(2009), no. 1, 3-21.

[35] A.Distler, Classification and Enumeration of Finite semigroups, $\mathrm{PhD}$ thesis, University of St. Andrews, 2010.

[36] A.Distler, M.Shah, V.Sorge, Enumeration of AG-groupoids, CICM 2011: Lecture Notes in Computer Science, vol. 6824, 1-14, 2011 DOI: 10.1007/978-3-642-22673-1_1

[37] Q.Mushtaq, M.Khan, Decomposition of $\mathrm{AG}^{*}$-groupoids, Quasigroups and related system, 15(2007), 303-308.

[38] W.A.Dudek, R.S.Gigon, Congruences on completely inverse $\mathrm{AG}^{* *}$-groupoids, Quasigroups and Related System, 20(2012), 203-209.

\title{
Перечень би-коммутативно-AG-группоидов
}

\author{
Мухаммед Рашад \\ Имтиаз Ахмад \\ Университет Малаканд \\ Чакдара, Пакистан \\ Мухаммед Шах \\ Правительственный колледж Пешавара \\ Пешавар, Пакистан \\ А. Боруманд Саид \\ Шахид Бахонарский университет \\ Кермане, Иран
}

\begin{abstract}
Аннотация. В этой статье мы вводим (слева, справа) бикоммутативные AG-группоиды и предлагаем простой метод проверки, является ли произвольный AG-группоид бикоммутативным AGгруппоидом или нет. Мы также исследуем некоторые общие свойства этих AG-группоидов. Далее вводим и изучаем некоторые свойства идеалов в этих AG-группоидах и разлагаем левые коммутативные AG-группоиды, определяя некоторые конгруэнции на этих AG-группоидах.
\end{abstract}

Ключевые слова: AG-группоид, бикоммутативно-AG-группоиды, (слева, справа) коммутативные AG-группоиды. 